\title{
The Colonization of Vancomycin-Resistant Enterococcus (VRE): Prevalence, Molecular Epidemiology, and Risk Factors in Patients Admitted to Intensive Care Units in Beijing.
}

\author{
Mengyao Yan \\ Peking University First Hospital \\ Yuanhui He \\ Peking University First Hospital

\section{Genjie Ruan} \\ Peking University First Hospital
}

\section{Feng Xue}

Peking University First Hospital

Bo Zheng ( $\nabla$ doctorzhengbo@163.com )

Peking University First Hospital https://orcid.org/0000-0002-6239-4047

Yuan LV

Peking University First Hospital

\section{Research}

Keywords: Vancomycin-resistant Enterococcus, Colonization, Molecular epidemiology, Intensive care patients, Risk factors

Posted Date: September 15th, 2021

DOI: https://doi.org/10.21203/rs.3.rs-880097/v1

License: (c) (i) This work is licensed under a Creative Commons Attribution 4.0 International License. Read Full License 


\section{Abstract}

Background: Vancomycin-resistant Enterococcus (VRE), as an important nosocomial pathogens, can be carried in gut for a long period and its colonization status associated with the subsequent infections. The aim of this study was to investigate the frequency of intestinal VRE colonization and identify the risk factors associated with VRE carriage in intensive care patients.

Methods: We conducted a 4-week cross-sectional study at six hospitals in Beijing, China. Patients admitted to Intensive Care Units (ICUs) were screened for intestinal colonization of VRE every Tuesday morning. Rectal swabs were selectively cultured for VRE, then the identified strains were analyzed by PCR to detect the glycopeptide resistance gene and were characterized by MLST. Risk factors were recorded to assess their effect on VRE acquisition during ICUs stay.

Results: Of 148 patients recruited, 46 (31.1\%) were colonized with VRE, with the majority ( $\mathrm{n}=42)$ being Enterococcus faecium. In total, $78.3 \%$ of the VRE were vanA positive and $15.2 \%$ vanM positive, while $6.5 \%$ undetected glycopeptide resistance gene. The predominant ST was ST78 (47.6\%) followed by ST192 (14.3\%), ST555 (9.5\%), ST789 (9.5\%), ST547 (4.8\%), and ST922 (4.8\%). Risk factors associated with VRE carriage were age of $>65$ years, a longer length of ICU stay, use of an endotracheal tube, and prior glycopeptides use.

Conclusions: The overall incidence proportion of VRE colonization at ICUs was relatively high in Beijing, and clonal expansion and horizontal transmission of resistant genes were both found here. Routine screening is necessary to prevent the dissemination of VRE.

\section{Background}

Enterococcus (including Enterococcus faecalis and Enterococcus faecium) is a prominent causes of healthcare-associated infections, and hospital-adapted lineages are increasingly resistant to vancomycin and widely disseminate $(1,2,3)$. In the United States, there are 54500 healthcare-associated infections caused by Vancomycin-resistant Enterococcus (VRE), resulting in 5400 deaths in $2017(4,5)$. The prevalence of VRE presents a huge challenge to infection control and treatment owing to their multidrug resistance and their propensity for persistence in the hospital environment (6). In particular, the Infectious Disease Society of America has listed E. faecium among the "no ESKAPE" pathogens (E. faecium, Staphylococcus aureus, Klebsiella pneumoniae, Acinetobacter baumannii, Pseudomonas aeruginosa, and Enterobacterspp.), for which there is an urgent need for new therapies (7).

The gastrointestinal (GI) tract is the primary reservoir for Enterococcus, which normally constitute a small proportion of the gut microbiota $(6,8)$. However, the exposure of hospitalized patients to antibiotics results in substantial changes in the gut microbiota that facilitate VRE colonization, especially among patients with critical illness in the intensive care units (ICUs) $(9,10,11)$. The process seems to be the important first step towards nosocomial enterococcal infections (6). Moreover, most patients from whom VRE are recovered are colonized rather than infected with the organism. Because the colonized patients 
are generally asymptomatic, the GI tract reservoir can easily go unnoticed unless surveillance culture specimens are obtained from patients at risk.

Despite VRE strains have increasingly been isolated from hospitalized patients in recent years, the prevalence of VRE in China is still relatively low. The China Antimicrobial Resistance Surveillance System (CARSS) reported that the national mean proportion of vancomycin-resistant $E$. faecium (VREfm) was $1.1 \%$ in 2019 (http://www.carss.cn/Report/Details?ald=770). Of note, the prevalence of VREfm in Beijing, the capital of China, was markedly higher with the isolation rate of $7.7 \%$. Despite the existing evidence, a comprehensive epidemiological picture of VRE in Beijing is lacking. We therefore conducted a multicenter study to assess the incidence proportion of VRE colonization in ICUs, and identified the risk factors associated with VRE acquisition.

\section{Methods}

\section{Study Design and Participants}

Over a 4-week period from 2 April to 1 May 2017, a cross-sectional study was conducted at six tertiarycare hospitals in Beijing, the capital of China. Adult patients ( $\geq 18$ years) were considered for the study if they were admitted to the ICUs during study period. More specifically, patients were eligible if they were either directly admitted to the ICUs or transferred to the ICUs from a hospital ward. In the participating ICUs, a rectal swab was performed on patients every Tuesday morning during this 4-week period to evaluate the VRE colonization status. Rectal swabs were collected by nurses with patients turned onto the left lateral decubitus position and the swab inserted deeply into the rectal canal and rotated 3 times. Swabs were transported in liquid Amies media for VRE culture and then flash-frozen at $-80^{\circ} \mathrm{C}$. Only the first eligible screening of VRE for each patient was considered for analysis if there were multiple clinical samples from the same patient. The study was approved by the Institutional Review Board of Peking University First Hospital (2020 Research 202). Written informed consent from the participants' legal guardian/next of kin was not required to participate in this study in accordance with the national legislation and the institutional requirements. In addition, a statement of permission from patients for submission was not required as the study did not include any personal information

\section{Epidemiologic and clinical data}

The patients' epidemiologic and clinical data collected included sociodemographics (age, gender, and ethnicity), comorbidity (cardiovascular disease, liver disease, renal disease, neoplasm, central nervous system disease, chronic pulmonary disease, and diabetes mellitus), prior exposure to medical device (central venous catheterization, endotracheal tube, and indwelling urinary catheter), the length of hospital stay at the time of screening, the length of ICU stay at the time of screening, antibiotics exposure in the preceding 7 days, a history of surgical procedures, and prior anti-cancer chemotherapies.

Data were obtained electronically from medical and pharmacy records maintained by the hospitals and then manually summarized in a paper-based questionnaire by the coordinating physicians. 


\section{VRE screening and identification}

The screening culture was performed by streaking a rectal swab in trilinear method onto an esculin agar medium containing vancomycin $(6 \mu \mathrm{g} / \mathrm{mL})$ followed by incubation at $37^{\circ} \mathrm{C}$ for 24 or $48 \mathrm{~h}$. Suspicious single colonies were transferred to blood plates containing vancomycin $(6 \mu \mathrm{g} / \mathrm{mL})$ for $24-48 \mathrm{~h}$ for purification culture. Then the bacterial isolates were identified by the Analytical Profile Index system and $16 \mathrm{~S}$ rRNA analysis. Total DNA as the template for polymerase chain reaction (PCR) was extracted from the bacterial isolates using DNA extraction Kit (Bacterial Genome DNA Extraction Kit; TIANGEN, Catalog no. DP 302) according to the manufacturer's guidelines (Bacterial Genome DNA Extraction Handbook DP190814) and stored at $-20^{\circ} \mathrm{C}$. PCR and sequencing were used to determine the presence of glycopeptide resistance genes $\operatorname{van} A, B, C, D, E, G, L, M$ and $N$ using a previously published protocol (12).

\section{Antimicrobial susceptibility testing}

MICs were determined by the agar dilution method (only daptomycin by the broth microdilution method) and interpretative breakpoint criteria were in accordance with those recommended by the Clinical and Laboratory Standards Institute (CLSI) guidelines $(13,14)$, with the exception of tigecycline, which was in accordance with the European Committee on Antimicrobial Susceptibility Testing (EUCAST) (15). A total of 14 antimicrobial agents were tested: ampicillin, vancomycin, teicoplanin, erythromycin, tetracycline, minocycline, tigecycline, ciprofloxacin, linezolid, levofloxacin, nitrofurantoin, rifampin, chloramphenicol, and daptomycin. Bacterial suspensions ( $>10^{4} \mathrm{CFU}$ of each bacterium) were obtained by a multipoint inoculator. E. faecalis ATCC 29212 was used as quality control reference strains.

\section{Molecular epidemiology}

E. faecium multilocus sequence typing (MLST) was conducted using previously published methods. Sequence types (STs) were determined using the MLST database (http://efaecium.mlst.net) and genetic relatedness was explored using BURST.

\section{Statistical approach}

We used frequencies and percentages for categorical variables and medians and interquartile ranges (IQR) for continuous variables for descriptive analyses. The differences in characteristics between VREcolonized and non-colonized patients were compared using the chi-square test or Fisher's exact test where appropriate. Differences in age and the duration of stay were compared using the Mann-Whitney $U$ test, as the data did not follow a normal distribution. All reported $P$ values were two-tailed with an $a$ level of 0.05. All data were analyzed using Statistical Package for the Social Sciences 26.0 software (SPSS, Inc., Chicago, IL, USA). Minimum spanning tree (MST) analysis was carried out using the BURST algorithm for related STs between different backgrounds by BioNumerics 7.5 software (Applied Maths, Belgium).

\section{Results}




\section{VRE prevalence in ICUs}

During the study period, from 2 April to 1 May 2017, a total of 162 patients were admitted to ICUs and they were all screened for rectal colonization with VRE. However, 14 patients were not incorporated into the study, as the related data of the 14 patients' medical records could not be retrieved in time. In the end, 148 patients' information were obtained for analysis.

Participants were predominantly male $(n=90 ; 60.8 \%)$, and the median age was 78 years (IQR, 62 to 83 years). Of the 148 participants, $46(31.1 \%)$ were colonized with VRE, the majority of which were identified as $E$. faecium $(n=42)$. PCR analysis revealed that the predominant gene cluster conferring vancomycin resistance among the tested VRE strains was the vanA cluster, present in $78.3 \%$ of strains $(\mathrm{n}=35)$, while $15.2 \%$ of strains $(n=8)$ harbored the vanM cluster and 3 strains (including 1 E. faecalis, 1 Enterococcus casseliflavus and 1 Enterococcus gallinarum) undetected genotypes. Table 1 summarizes the patients' characteristics. 
Table 1

Characteristics of patients and their colonized vancomycin-resistant enterococci ${ }^{\mathrm{a}}$

\section{Characteristics}

Age (yr)

Median (IQR)

Range

No. $(\%)$ of patients $>65$ year of age

No. (\%) of patients by gender

Male

Female

No. (\%) of patients screened for VRE in the following hospital:

Hospital A

Hospital B

Hospital C

Hospital D

Hospital E

Hospital F

No. (\%) of patients colonized with VRE

No. (\%) of patients with VRE of the following type:

Enterococcus faecium

Other

No. (\%) of patients with VRE of the following genotype:

vanA cluster

vanM cluster

a Abbreviations: IQR, interquartile range; VRE, vancomycin-resistant enterococci.
Values for patients $(n=148)$

$78(62-83)$

25-95

$101(68.2)$

90 (60.8)

58 (39.2)

39 (26.4)

38 (25.7)

$34(23.0)$

15 (10.1)

$14(9.5)$

8 (5.4)

$46(31.1)$

$42(91.3)$

$4(8.7)$

36 (78.3)

$7(15.2)$

The prevalence of VRE colonization in six hospitals was 15.4\% (6/39), 21.1\% (8/38), 14.7\% (5/34), 80.0\% (12/15), 85.7\% (12/14), and 37.5\% (3/8), respectively. Apart from hospital D, vanM-type VRE were detected in all centers over the study period. There was no VRE outbreak in these six hospitals during the study period. 
A total of 42 VRE fm strains were available for antimicrobial susceptibility testing. All of the 42 strains displayed high levels of resistance to vancomycin, with MICs in the range of 64 to $>512 \mu \mathrm{g} / \mathrm{ml}$. Resistance was found for teicoplanin (50\%), ampicillin (100\%), erythromycin (83.3\%), tetracycline (57.1\%), minocycline (31.0\%), ciprofloxacin (100\%), levofloxacin (100\%), nitrofurantoin (59.5\%), rifampin (95.2\%), chloramphenicol (2.4\%), linezolid (0\%), daptomycin ( $0 \%)$, and tigecycline ( $0 \%)$. Detailed susceptibilities of VRE strains results are shown in Table 2.

Table 2

Susceptibilities of colonized vancomycin-resistant Enterococcus faecium strains to antimicrobial agents ${ }^{a}$

\begin{tabular}{|lllll|}
\hline Antibacterial agent & \multicolumn{1}{l}{ MIC $(\boldsymbol{\mu g} / \mathrm{ml})(\mathbf{n}=\mathbf{4 2})$ : } & & \\
\cline { 2 - 5 } & Range & MIC $_{50}$ & MIC $_{90}$ & $\mathbf{R}(\%)$ \\
\hline Vancomycin & 64 to $>512$ & 256 & 512 & 100 \\
\hline Teicoplanin & 0.25 to 128 & 16 & 64 & 50 \\
\hline Linezolid & 1 to 4 & 1 & 2 & 0 \\
\hline Daptomycin & 0.062 to 2 & 1 & 2 & 0 \\
\hline Tigecycline & 0.015 to 0.125 & 0.062 & 0.125 & 0 b \\
\hline Ampicillin & 128 to $>512$ & 512 & $>512$ & 100 \\
\hline Erythromycin & $<0.062$ to $>512$ & 512 & 512 & 83.3 \\
\hline Tetracycline & 0.25 to 256 & 32 & 128 & 57.1 \\
\hline Minocycline & 0.031 to 32 & 8 & 16 & 31.0 \\
\hline Ciprofloxacin & 16 to $>512$ & 128 & 512 & 100 \\
\hline Levofloxacin & 32 to 128 & 64 & 128 & 100 \\
\hline Nitrofurantoin & 32 to 512 & 128 & 256 & 59.5 \\
\hline Rifampin & $<0.062$ to 64 & 16 & 32 & 95.2 \\
\hline Chloramphenicol & 4 to 64 & 8 & 16 & 2.4 \\
\hline a CLSI 2020 breakpoints were applied except tigecycline. & \\
\hline b ECusT 2020 breakpoints were applied. & & & \\
\hline
\end{tabular}


Table 3

Univariate analysis of epidemiologic and clinical risk factors for vancomycin-resistant enterococci colonization $^{\mathrm{a}}$

Characteristic
VRE positive VRE negative $P$ $(n=46$;

$31.1 \%)$ $(n=102$;

$68.9 \%)$

\section{Demographics}

Median (IQR) age (years)

$83(76-86) \quad 72(61-83)$

$0.001^{\mathrm{b}}$

No. $(\%)$ of patients $>65$ years of age

$39(84.8)$

$62(60.8)$

0.004

No. (\%) of male patients

$31(67.4)$

$59(57.8)$

0.271

Details of hospital stay

Median (IQR) length of hospital stay (days)

$15(8.5-29)$

$11.5(5-27)$

0.179

Median (IQR) length of ICU stay (days)

$13.5(6-26)$

$7.5(1-26)$

0.039

Comorbidities

No. (\%) of patients with the following:

Cardiovascular disease

$12(26.1)$

$18(17.6)$

0.237

Chronic pulmonary disease

9 (19.6)

$24(23.5)$

0.592

Hepatic dysfunction

$3(6.5)$

$1(0.9)$

$0.089^{c}$

Renal dysfunction

$7(15.2)$

$7(6.7)$

$0.132^{\mathrm{C}}$

No. (\%) of patients with the following medical devices:

Central venous catheterization

$21(45.7)$

$36(35.3)$

0.231

Endotracheal tube

$21(45.7)$

$24(23.5)$

0.007

Indwelling urinary catheter

$35(76.1)$

$66(64.7)$

0.169

No. (\%) patients with the following type of antibiotic therapy:

Cephalosporins

$17(37.0)$

$33(32.4)$

0.584

Carbapenems

$14(30.4)$

39 (38.2)

0.360

a Abbreviations: IQR, interquartile range.

b Mann-Whitney U test.

${ }^{\mathrm{c}}$ Fisher's exact test. 


\begin{tabular}{|llll|}
\hline Characteristic & $\begin{array}{l}\text { VRE positive } \\
(\mathbf{n = 4 6 ;}\end{array}$ & $\begin{array}{l}\text { VRE negative } \\
(\mathbf{n = 1 0 2 ;} \\
\mathbf{6 8 . 9 \% )}\end{array}$ & $P$ \\
\hline Glycopeptides & $7(15.2)$ & $5(4.9)$ & $0.049^{\mathrm{c}}$ \\
\hline Fluoroquinolones & $5(10.9)$ & $6(5.9)$ & $0.318^{\mathrm{c}}$ \\
\hline Linezolids & $4(8.7)$ & $9(8.8)$ & $1.000^{\mathrm{c}}$ \\
\hline a Abbreviations: IQR, interquartile range. & & & \\
\hline b Mann-Whitney U test. & & & \\
\hline c Fisher's exact test. & & & \\
\hline
\end{tabular}

\section{Genetic distribution of isolates}

Using seven-locus MLST, all VREfm strains were grouped into 10 STs. The distribution of STs over the study period is depicted in Fig. 1. The predominant ST among the 42 strains was ST78 $(n=20,47.6 \%)$. ST192 $(n=6,14.3 \%)$ was the second most predominant ST. In addition, ST555 and ST789 accounted for four strains (9.5\%) each. ST547 and ST922 accounted for two strains (4.8\%) each. Finally, four strains were singletons belonging to ST17, ST80, ST343, and ST389, respectively.

\section{Patient factors associated with the VRE colonization}

Risk factors were compared between the group of VRE-positive patients and all patients being not VREcolonized. Univariate analysis revealed that significant risk factors associated with becoming a carrier patient included age of $>65$ years, a longer length of ICU stay, use of an endotracheal tube, and exposures to glycopeptides in the preceding 7 days.

\section{Discussion}

Enterococcus, a globally important opportunistic pathogen, can be carried in the Gl tract for a long period without any symptoms of infection and likewise persist in the hospital environment (16). Enterococcus is inherently resistant to a number of antimicrobial classes, and over recent decades there has been a significant increase in the rates of acquired antimicrobial resistance (AMR) in E. faecalis and E. faecium, including VRE $(17,18)$. Previous studies have demonstrated that enterococcus status at ICU admission was associated with risk for death or all-cause infection, and the gastrointestinal microbiome may have a role in risk stratification and early diagnosis of ICU infections (11). There is a need for active surveillance to better prevent the emergence and dissemination of VRE. 
We performed a multicenter study investigating the prevalence of gastrointestinal colonization with VRE in hospital patients in Beijing and analyzing the molecular epidemiology of VRE. Although this study was performed on a selection of hospital patients, i.e., patients admitted to ICUs, the results are of critical importance since these patients are especially prone to colonization and (subsequent) infection. We reported a mean proportion of $31.1 \%$ (46 of 148 ) for culture positive intestinal carriage of VRE in ICU, and found that vanA-type VRE and vanM-type VRE were both detected in almost every hospital but vanA-type was more popular. We additionally identified age, longer ICU stay, use of an endotracheal tube, and prior glycopeptides exposure as significant risk factors. To our knowledge, this is the largest study investigating the prevalence of VRE colonization in ICU in China mainland, and the results are further strengthened by the multicenter design and comprehensive capture of potential risk factors through a standardized medical record review.

Several previous studies have reported a prevalence of VRE carriage on ICU admission ranging between $2.5 \%$ and $40 \%(19,20,21)$. In this study, we found the overall prevalence of VRE carriage at ICU was markedly high. Moreover, there were huge difference in the isolation rates of VRE among the six hospitals, and the lowest isolation rate was $14.7 \%$ (5/34) but the highest rate up to $85.7 \%(12 / 14)$. Hospital variations are not unusual for drug-resistant bacteria, as different compliance with isolation practices, infection control, and antibiotic stewardship programs that affect behavior among the health care personnel, account for this differences. In addition, the variations may be related to the patients' characteristics prior to ICU admission (surgical wards, transplantation units, etc.), as well as, the limited sample size in part of the hospitals $(22,23)$. Altogether, the incidence proportion of VRE colonization was comparatively high in Beijing and might be neglected before. Previous studies have indicated that pathogens can be cultured from stool or swabs that predict specific infections, and VRE colonization has been established as a risk factor for subsequent infection. Our study results might strengthen the need for prompt identification of colonized patients and to carry out the effective multifaceted strategies to control VRE in healthcare institution.

E. faecium (42/46) predominated among colonizing VRE in this study, as reported elsewhere. A high level of glycopeptide resistance is more common in E. faecium than $E$. faecalis (24). In addition, $E$. casseliflavus accounted for two VRE strains, and another two VRE were singletons belonging to $E$. faecalis, and E. gallinarum, respectively. Among the tested VRE, 35 strains harbored vanA resistance gene and 8 strains carried van $M$ gene, and there was no van $B$ gene detected. Glycopeptide resistance in enterococci is mediated by van gene clusters, among which vanA and $\operatorname{van} B$ are the most commonly reported worldwide $(25,26,27,28)$. In 2006 , vanM was first reported as a new and prevalent resistance determinant in clinical enterococci in China (29). Subsequently, vanM-type VRE has spread rapidly around the country, especially in the cities of Shanghai and Hangzhou $(24,30)$. In 2011, a study reported a vanMtype $E$. faecium clinical strain was detected in Singapore, which indicated that vanM gene might spread to other countries (31). Epidemiology data for strains with vanM cluster remain rare in Beijing, and previous results were limited to single-center investigations. In this study, vanM-type VRE were detected in almost every participating hospital, thus suggesting that vanM cluster plays an important role in 
vancomycin resistance and van gene clusters dissemination in Beijing. However, our study showed that vanA cluster still was the dominant resistance determinant in enterococcus in Beijing.

The tested E. faecium, including vanA-type strains and vanM-type strains, all displayed the high vancomycin MIC values. Interestingly, these strains showed variable levels of resistance to teicoplanin, with the MIC ranged from 0.25 to $128 \mu \mathrm{g} / \mathrm{ml}$. Although the strains carried the same type of van cluster, they displayed the different susceptibilities to teicoplanin. This phenotype variation may to some degree be related to the integrality of the van operon $(32,33)$. Most of the colonization VRE were resistant to several kind of antimicrobial agents, and they belong to multi-drug resistant (MDR) (resistant to three or more antimicrobial classes). Fortunately, linezolid, daptomycin, and tigecycline demonstrated complete in vitro activity against these strains.

MLST typing displayed two dominant STs, including ST78, and ST192, which were frequently identified in VREfm strains in China, and the most common ST78 occurred in each participating hospital $(34,35,36)$. All but one E. faecium strain (ST922) in this study belonged to clonal complexes (CC) 17, which represents a lineage of a virulent VRE hospital clone that has been observed worldwide (2, 37-42). The results described here clearly suggest a clonal spread of the highly adapted and resistant lineage CC17 of E. faecium strains among hospitals. In addition, two E. faecium strains both carrying vanM resistance gene belonged to ST922, and they were detected in the same hospital. The fact that ST922 was not included in CC17, and no previous study has found ST922 strains carrying vanM cluster in China, goes some way to suggest a horizontal transfer of van cluster among $E$. faecium strains. Overall, these data indicate that clonal expansion and horizontal transfer of resistance genes have contributed to VRE increased prevalence in hospital.

This study identified four risk factors for VRE colonization at ICU: age, length of ICU stay, endotracheal tube use, and recent glycopeptides use, all of which agrees with the results of numerous other studies. Here, the attributed risk of VRE acquisition after glycopeptide treatment needs to be mentioned. Several previous studies have demonstrated that vancomycin might influence the likelihood of VRE colonization and transmission between patients. This phenomenon may be attributed to vancomycin being a narrowspectrum antibiotic and prescribed generally with other antibiotics, such as cephalosporins, and carbapenems, which disrupt the normal gut flora, thus the likelihood of VRE colonization may have increased. Another possible reason is that part of gut enterococci belong to vancomycin-variable enterococci (VVE), which are a group of enterococci containing van resistant genes that exhibit a vancomycin-susceptible phenotype but are capable of shifting to a glycopeptide-resistant phenotype under vancomycin therapy, as previously stated $(32,33)$.

The study has some limitations. First, the study period was relatively short, and thus the population was not large enough, which may influence the determination of risk factors for VRE colonization. Second, the rectal surveillance swab was not applied on patients at the time of admission but every Tuesday morning during the study period, which can lead the VRE colonization status of some patients unclear. Another limitation is the problem of screening process. We evaluated the VRE colonization status by way of 
phenotype identification, however, some patients colonized by VVE were neglected and the prevalence of van resistant gene might be underestimated.

\section{Conclusion}

The overall incidence proportion of VRE colonization at ICUs was relatively high in Beijing. Clonal expansion and horizontal transmission from other patients were both found in VRE strains. Four factors, including age older than 65 years, length of ICU stay, endotracheal tube use, and recent glycopeptides use, were associated with the VRE acquisition. As a result of our findings, we suggest that routine screening is necessary to prevent the dissemination of VRE in Beijing.

\section{Abbreviations}

VRE: Vancomycin-resistant Enterococcus; ICUs: Intensive care units; Gl: Gastrointestinal; CARSS: China Antimicrobial Resistance Surveillance System; VREfm: Vancomycin-resistant E. faecium; PCR:

Polymerase chain reaction; CLSI: Clinical and Laboratory Standards Institute; EUCAST: European Committee on Antimicrobial Susceptibility Testing; MLST: Multilocus sequence typing; STs: Sequence types; IQR: Interquartile range; MST: Minimum spanning tree; AMR: Antimicrobial resistance; MDR: Multidrug resistant; CC: Clonal complexes; VVE: Vancomycin-variable enterococci.

\section{Declarations}

\section{Acknowledgments}

We thank the patients who participated in this study and the staff who facilitated the study conduct and data collection.

\section{Informed consent}

Written informed consent from the participants' legal guardian/next of kin was not required to participate in this study in accordance with the national legislation and the institutional requirements.

\section{Authors' contributions}

MY, YH, GR, YL and BZ conceived the study design. MY, YH and GR were responsible for the recruitment and collection of samples. MY, YH, RG and FX were responsible for the laboratory assays. MY performed the data analysis and completed the initial manuscript. MY, $\mathrm{YH}, \mathrm{YL}$ and $\mathrm{BZ}$ revised the manuscript. All the authors read and approved the final version of the manuscript.

\section{Funding}

This work was supported by Beijing Municipal Science and Technology Commission (Grant No. Z161100000516040) and the National Key Research and Development Program of China 
(2018YFC1200100 and 2018YFC1200105).

\section{Availability of data and materials}

All data generated or analyzed during this study are included in this manuscript.

\section{Ethics approval and consent to participate}

The study was approved by the Institutional Review Board of Peking University First Hospital (2020 Research 202). We make sure to keep patient data confidential and compliance with the Declaration of Helsinki.

\section{Consent for publication}

Not applicable.

\section{Competing interests}

The authors declare that they have no competing interests.

\section{References}

1. Cetinkaya Y, Falk P, Mayhall CG. Vancomycin-resistant enterococci. Clin Microbiol Rev. 2000;13:686707.

2. De Oliveira DMP, Forde BM, Kidd TJ, Harris PNA, Schembri MA, Beatson SA et al. Antimicrobial resistance in ESKAPE pathogens. Clin Microbiol Rev. 2020;33:e00181-19.

3. Lebreton F, van Schaik W, McGuire AM, Godfrey P, Griggs A, Mazumdar V et al. Emergence of epidemic multidrug-resistant Enterococcus faecium from animal and commensal strains. mBio. 2013;4:e00534-13.

4. CDC's Antibiotic Resistance Threats in the United States, 2019. Centers for Disease Control and Prevention, US Department of Health andHuman

Services. https://www.cdc.gov/drugresistance/biggest-threats.html\#van Accessed May 17, 2021.

5. Zhou Y, Yang Y, Ding L, Chen C, Xu X, Wang M. Vancomycin Heteroresistance in vanM-type Enterococcus faecium. Microb Drug Resist. 2020;26:776-782.

6. Arias CA, Murray BE. The rise of the Enterococcus: beyond vancomycin resistance. Nat Rev Microbiol. 2012;10:266-78.

7. World Health Organization. Global priority list of antibioticresistant bacteria to guide research, discovery, and development of new antibiotics. 2017. http://www.who.int/medicines/publications/WHO-PPL-Short_Summary_25FebET_NM_WHO.pdf?ua\%1 Accessed May 17, 2021.

8. Eckburg PB, Bik EM, Bernstein CN, Purdom E, Dethlefsen L, Sargent M et al. Diversity of the human intestinal microbial flora. Science. 2005;308:1635-8. 
9. Donskey CJ, Chowdhry TK, Hecker MT, Hoyen CK, Hanrahan JA, Hujer AM et al. Effect of antibiotic therapy on the density of vancomycin-resistant enterococci in the stool of colonized patients. N Engl J Med. 2000;343:1925-32.

10. Ubeda C, Taur Y, Jenq RR, Equinda MJ, Son T, Samstein M et al. Vancomycin-resistant Enterococcus domination of intestinal microbiota is enabled by antibiotic treatment in mice and precedes bloodstream invasion in humans. J Clin Invest. 2010;120:4332-41.

11. Freedberg DE, Zhou MJ, Cohen ME, Annavajhala MK, Khan S, Moscoso D et al. Pathogen colonization of the gastrointestinal microbiome at intensive care unit admission and risk for subsequent death or infection. Intensive Care Med. 2018;44:1203-1211.

12. He Y, Ruan G, Hao H, Xue F, Zhu S, Xiao B et al. Evaluation of Quadruple Real-Time PCR Method to Detect Enterococci Carrying Vancomycin-Resistant Genes vanA, vanB, vanM in Rectal Swabs. Front Med. 2020;7:403.

13. CLSI. Methods for dilution antimicrobial susceptibility tests for bacteria that grow aerobically; approved standard, 9th ed. CLSI document M07-A9. 2012.

14. CLSI. Performance standards for antimicrobial susceptibility testing, 30th ed. CLSI document M100Ed30. 2020.

15. EUCAST. Breakpoint Tables for Interpretation of MICs and Zone Diameters, Version 8.0, 2020.

16. Lin MY, Hayden MK. Methicillin-resistant Staphylococcus aureus and vancomycin-resistant enterococcus: Recognition and prevention in intensive care units. Crit Care Med. 2010;38:S335-44.

17. Gorrie C, Higgs C, Carter C, Stinear TP, Howden B. Genomics of vancomycin-resistant Enterococcus faecium. Microb Genom. 2019;5:e000283.

18. Deshpande LM, Fritsche TR, Moet GJ, Biedenbach DJ, Jones RN. Antimicrobial resistance and molecular epidemiology of vancomycin-resistant enterococci from North America and Europe: a report from the SENTRY antimicrobial surveillance program. Diagn Microbiol Infect Dis. 2007;58:163170.

19. Yoon YK, Kim HJ, Lee WJ, Lee SE, Yang KS, Park DW et al. Clinical prediction rule for identifying patients with vancomycin-resistant enterococci (VRE) at the time of admission to the intensive care unit in a low VRE prevalence setting. J Antimicrob Chemother. 2012;67:2963-9.

20. Morgan DJ, Day HR, Furuno JP, Young A, Johnson JK, Bradham DD et al. Improving efficiency in active surveillance for methicillin-resistant Staphylococcus aureus or vancomycin-resistant Enterococcus at hospital admission. Infect Control Hosp Epidemiol. 2010;31:1230-5.

21. Minhas P, Perl TM, Carroll KC, Shepard JW, Shangraw KA, Fellerman D et al. Risk factors for positive admission surveillance cultures for methicillin-resistant Staphylococcus aureus and vancomycinresistant enterococci in a neurocritical care unit. Crit Care Med. 2011;39:2322-9.

22. Papadimitriou-Olivgeris M, Drougka E, Fligou F, Kolonitsiou F, Liakopoulos A, Dodou V et al. Risk factors for enterococcal infection and colonization by vancomycin-resistant enterococci in critically ill patients. Infection. 2014;42:1013-22. 
23. Ziakas PD, Thapa R, Rice LB, Mylonakis E. Trends and significance of VRE colonization in the ICU: a meta-analysis of published studies. PLoS One. 2013;8:e75658.

24. Huang L, Zhang R, Hu Y, Zhou H, Cao J, Lv H et al. Epidemiology and risk factors of methicillinresistant Staphylococcus aureus and vancomycin-resistant enterococci infections in Zhejiang China from 2015 to 2017. Antimicrob Resist Infect Control. 2019;8:90.

25. Hammerum AM, Baig S, Kamel Y, Roer L, Pinholt M, Gumpert $\mathrm{H}$ et al. 2017. Emergence of vanA Enterococcus faecium in Denmark, 2005-15. J Antimicrob Chemother. 2017;72:2184-2190.

26. Asgin N, Otlu B. Antibiotic Resistance and Molecular Epidemiology of Vancomycin-Resistant Enterococci in a Tertiary Care Hospital in Turkey. Infect Drug Resist. 2020;13:191-198.

27. Bourdon N, Fines-Guyon M, Thiolet JM, Maugat S, Coignard B, Leclercq R et al. Changing trends in vancomycin-resistant enterococci in French hospitals, 2001-08. J Antimicrob Chemother. 2011;66:713-21.

28. Freitas AR, Tedim AP, Francia MV, Jensen LB, Novais C, Peixe L et al. Multilevel population genetic analysis of vanA and vanB Enterococcus faecium causing nosocomial outbreaks in 27 countries (1986-2012). J Antimicrob Chemother. 2016;71:3351-3366.

29. Xu X, Lin D, Yan G, Ye X, Wu S, Guo Y et al. vanM, a new glycopeptide resistance gene cluster found in Enterococcus faecium. Antimicrob Agents Chemother. 2010;54:4643-7.

30. Chen C, Sun J, Guo Y, Lin D, Guo Q, Hu F et al. High Prevalence of vanM in Vancomycin-Resistant Enterococcus faecium Isolates from Shanghai, China. Antimicrob Agents Chemother. 2015;59:77958.

31. Teo JWP, Krishnan P, Jureen R, Lin RTP. Detection of an Unusual van Genotype in a VancomycinResistant Enterococcus faecium Hospital Isolate. J Clin Microbiol. 2011;49:4297-8.

32. Sun L, Qu T, Wang D, Chen Y, Fu Y, Yang Q et al. Characterization of vanM carrying clinical Enterococcus isolates and diversity of the suppressed vanM gene cluster. Infect Genet Evol. 2019;68:145-152.

33. Sun L, Chen Y, Hua X, Chen Y, Hong J, Wu X et al. Tandem amplification of the vanM gene cluster drives vancomycin resistance in vancomycin-variable enterococci. J Antimicrob Chemother. 2020;75:283-291.

34. Sun H, Liu C, Zhang J, Zhou Y, Xu Y. Molecular characterization of vancomycin-resistant enterococci isolated from a hospital in Beijing, China. J Microbiol Immunol Infect. 2019;52:433-442.

35. Yang J, Li T, Ning Y, Shao D, Liu J, Wang S et al. Molecular characterization of resistance, virulence and clonality in vancomycin-resistant Enterococcus faecium and Enterococcus faecalis: A hospitalbased study in Beijing, China. Infect Genet Evol. 2015;33:253-60.

36. Yang J, Jiang Y, Guo L, Ye L, Ma Y, Luo Y. Prevalence of Diverse Clones of Vancomycin-Resistant Enterococcus faecium ST78 in a Chinese Hospital. Microb Drug Resist. 2016;22:294-300.

37. Lee T, Pang S, Abraham S, Coombs GW. Antimicrobial-resistant CC17 Enterococcus faecium: the past, the present and the future. J Glob Antimicrob Resist. 2019;16:36-47. 
38. Coombs GW, Pearson JC, Daley DA, Le T, Robinson OJ, Gottlieb T et al. Molecular epidemiology of enterococcal bacteremia in Australia. J Clin Microbiol. 2014;52:897-905.

39. da Silva LP, Pitondo-Silva A, Martinez R, da Costa Darini AL. Genetic features and molecular epidemiology of Enterococcus faecium isolated in two university hospitals in Brazil. Diagn Microbiol Infect Dis. 2012;74:267-271.

40. Kang M, Xie Y, He C, Chen ZX, Guo L, Yang Q et al. Molecular characteristics of vancomycin-resistant Enterococcus faecium from a tertiary care hospital in Chengdu, China: molecular characteristics of VRE in China. Eur J Clin Microbiol Infect Dis. 2014;33:933-939.

41. Strateva T, Sirakov I, Dimov S, Trifonova A, Savov E, Mitov I. Clonal spread of vanA Enterococcus faecium sequence type 203 in Bulgarian hospitals. Infect Dis (Lond). 2018;50:718-721.

42. Gao W, Howden BP, Stinear TP. Evolution of virulence in Enterococcus faecium, a hospital-adapted opportunistic pathogen. Curr Opin Microbiol. 2018;41:76-82.

\section{Figures}




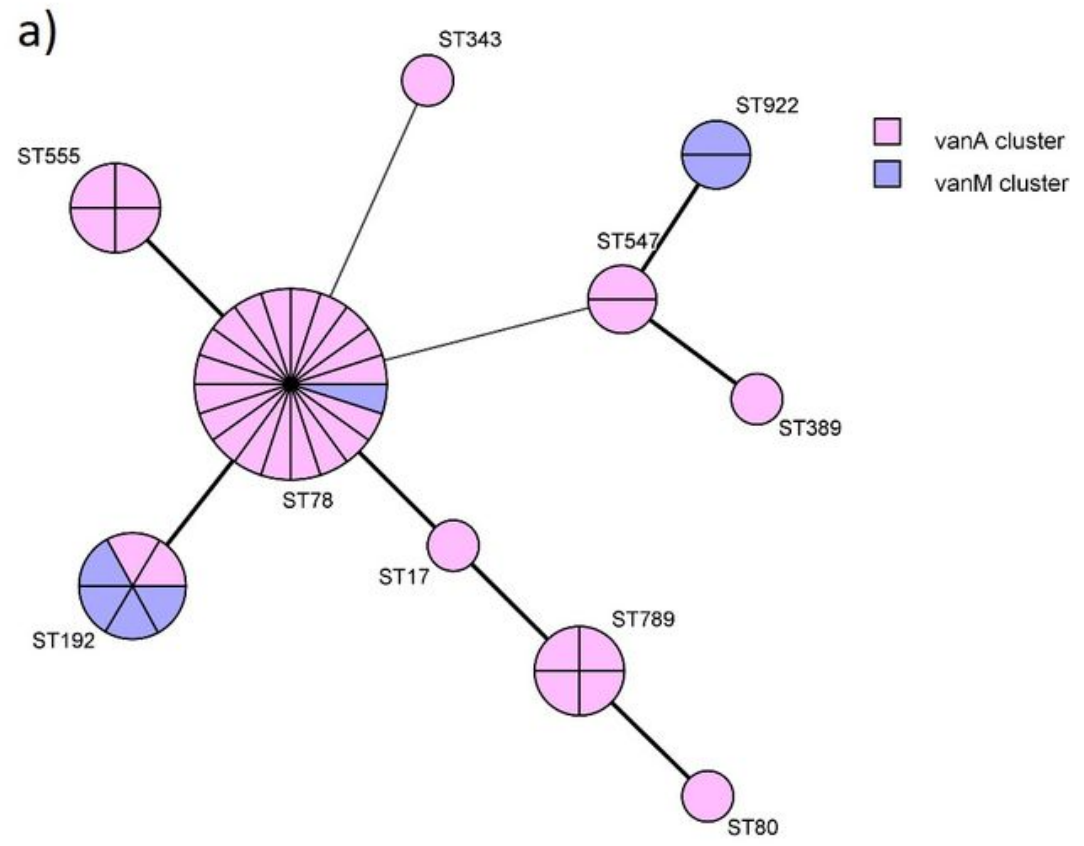

b)

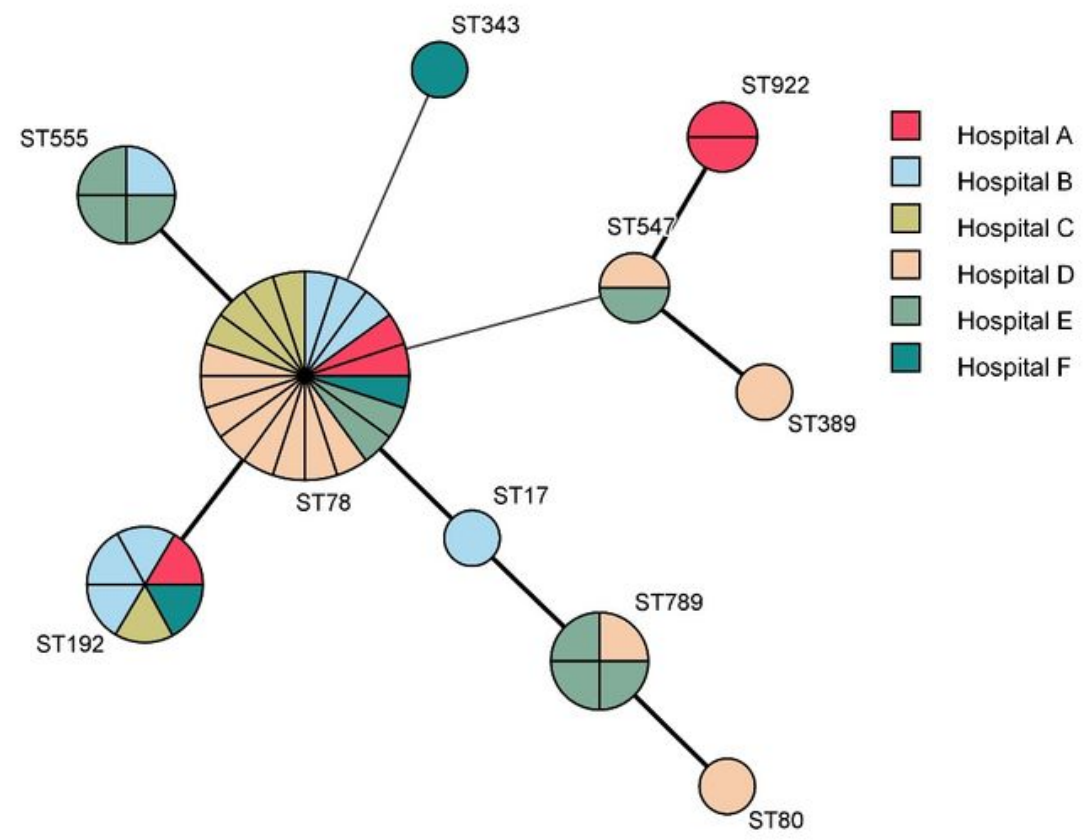

\section{Figure 1}

MST analysis of the 42 vancomycin-resistant Enterococcus faecium strains generated from MLST data. Each ST is displayed as a circle and the size of the circle denotes the number of strains belonging to that particular ST. Lengths of lines between each circle/ST proportionally demonstrate the number of different alleles. Each circle is labelled with the corresponding ST. Different groups of strains are identified by 
different colours. Strains were divided into two groups according to van genotype (a), and six groups according to hospital of isolation (b). 